\title{
Preisdurchsetzung im Service: Zahlungsbereitschaft durch Nutzenargumentation
}

\author{
Thomas Biermann
}

\section{Kundenorientierung - kein Selbstzweck}

Kundenorientierung, Servicequalität und Innovation das sind die zentralen Felder der Herausforderung für ein modernes Unternehmen. Aber wer soll das bezahlen? Erweiterte Produktfunktionalitäten, längere Lebensdauer, individuelle Beratung, 24-Stunden-Verfügbarkeit der Service-Hotline, uneingeschränkte Geld-zurückGarantien - all das ist aus Kundensicht ohne Zweifel angenehm. Nur rechnet sich das auch fuir das Unternehmen? Die konsequente Ausrichtung des Unternehmens auf die Erfüllung von Kundenwünschen wird erst dann zur betriebswirtschaftlich zielfuihrenden Strategie, wenn genügend Abnehmer die erbrachten Leistungen durch entsprechende Zahlungen honorieren. Ohne ein Service Controlling wird übertriebenes Service Marketing leicht zum Ergebniskiller (Biermann 1999).

\section{Service Marketing und Service Controlling}

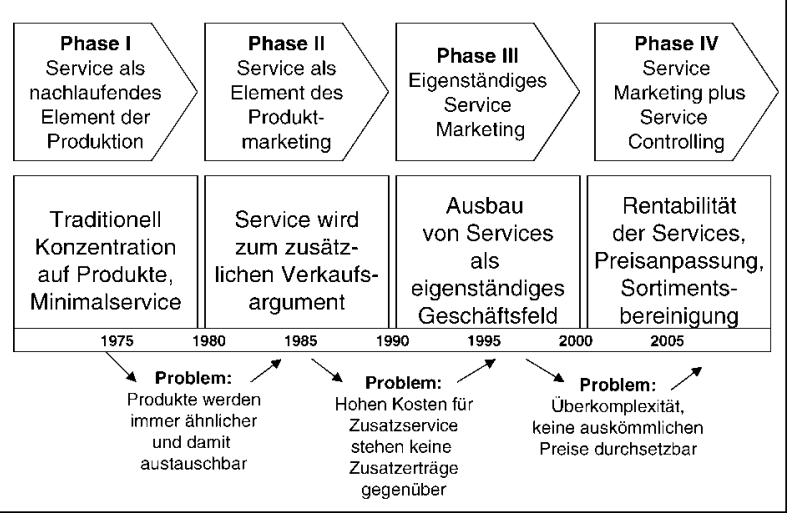

Abb. 1: Service Marketing und Service Controlling

Wir wollen am Beispiel von Serviceleistungen beleuchten, wie die Durchsetzung auskömmlicher Preise im Markt realisiert werden kann. Dabei nutzen wir Erfahrungen aus Projekten in der Logistik, bei Banken und im Bereich technischer Dienstleistungen im Automobilsektor. Industriebetriebe haben ihre Dienstleistungspalette in den vergangenen Jahren stark ausgeweitet. Sie vertrauen auf Langfristeffekte der Kundentreue, die - so hoffen sie die teuren Maßnahmen der Serviceverbesserung eines Tages finanzieren werden. Es wird jedoch darauf ankommen, die Kräfte auf diejenigen Aktivitäten zu konzentrieren, bei denen nachweislich eine hohe Hebelwirkung zwischen Aufwand und späterem Ertrag besteht. Vor dem bedenkenlosen Verschenken von vielfältigen Zusatzleistungen ohne Kenntnis der Wertschätzung durch den Kunden ist zu warnen - eine Serviceüberschwemmung ist aus betriebswirtschaftlicher Sicht mindestens ebenso gefährlich wie die gern bejammerte Servicewüste.

\section{Nutzenargumentation im Wettbewerb}

\subsection{Wer bestellt, bezahlt}

Die Hoffnung auf einen Wettbewerbsvorsprung durch Weiterdrehen der Serviceschraube geht ins Leere, wenn alle Konkurrenten den gleichen Weg verfolgen und ihr Serviceangebot kontinuierlich verbessern. Trotz erhöhter Kosten hat man seine relative Position nur gerade behauptet. Ein Hol- und Bringservice seitens der Autowerkstatt beispielsweise, vor wenigen Jahren noch ein echtes Service-Schmankerl, ist heute im Premiumsegment bereits zum Standard geworden.

Auf längere Sicht rechtfertigt sich eine Investition in Infrastrukturen für Top-Service nur durch die Erwartung, mit den Dienstleistungen selbst Geld zu verdienen und aus ihnen eigene Geschäftsfelder zu entwickeln. Wer unprofitable Leistungsangebote im Programm hält, weil er sich Synergieeffekte und Quersubventionierungen mit anderen Geschäftszweigen verspricht, kann wohl lange warten, bis er auf seine Kosten kommt. Ein Negativbeispiel bildet das Schicksal vieler Dienstleister im Internet: Es ist keine Kunst, mit kostenlosen Angeboten viele Abnehmer zu gewinnen, aber auf Leistungsgeschenken allein lässt sich kein funktionierendes Geschäftsmodell aufbauen. Heute glaubt selbst bei den pflichtoptimistischen Start-Ups niemand mehr, dass eines Tages ein wohltätiger Dritter für Anbieter und Kunden die Zeche zahlt - etwa mittels Werbeanzeigen in kostenlosen Internet-Services.

Es gilt also, vorrangig das Angebot von solchen Services zu forcieren, die der Kunde so schätzt, dass er für sie auch bezahlt - direkt oder indirekt. Im Umkehrschluss sollte man sich von Dienstleistungen verabschieden, bei denen die kundenseitige Wertschätzung auf Dauer nicht von entsprechender Zahlungsbereitschaft gestuitzt wird. Deshalb ist verstärkte Aufmerksamkeit auf die Frage zu richten, wie die Wertschätzung des Kunden entsteht und wie sie sich positiv beeinflussen lässt. Wo liegt der Kundennutzen? Was spart der Kunde an Geld, Zeit oder Unbequemlichkeit, wenn er unsere Services nutzt? Welche Alternativen hat er? Hilft ihm unsere Dienstleistung vielleicht, seinerseits Kunden zu gewinnen oder zu halten?

\subsection{Servicequalität im Zwiespalt}

Nur zu liefern, was der Kunde auch bezahlt, mit dieser Forderung tut sich das traditionelle Dienstleistungsgewerbe schwer, darin liegt sein Charme und sein wirtschaftliches Kernproblem. Der goldbetresste Husar vor 
dem Hotelportal bereichert ohne Zweifel das Stadtbild, aber kommt deswegen ein Gast mehr? Die Gute-NachtSchokolade auf dem Kopfkissen und das aufwendige Duftwasser-Set im Bad ist nett als Geste des Hauses, aber natuirlich keinesfalls kostenlos - das ist im Zimmerpreis einkalkuliert. Dass der freundliche Bankberater zwanzig Minuten Zeit opfert, mir den Kauf dieser abenteuerlichen Silberaktien auszureden, nimmt mich sehr für ihn ein wenn er bloß nicht gleich darauf den Prospekt des bankeigenen Immobilienfonds herausgezogen hätte.

Auf der anderen Seite erweist sich eine auf Einzelleistungen aufgebaute Aufpreispolitik, wie sie die Automobilhersteller bei Schiebedächern und Ledersitzen praktizieren, im Servicesektor als undurchfuihrbar. Das Scheitern des Pay-TV-Konzepts in Deutschland ist das aktuellste Beispiel, nicht das einzige: Der Versuch der Banken, sich zeitraubende Beratungsleistungen bei der Baufinanzierung separat honorieren zu lassen, wurde abgebrochen; desgleichen der Ansatz großer Reisebüros, für Informationen Entgelte zu erheben, wenn der Interessent keine Reise bei ihnen bucht. Kaum ein Internet-Anbieter hat es geschafft, für bisher kostenfreie Services später Entgelte zu verlangen. Wir müssen akzeptieren, dass im Auge des Kunden Dienstleistungen im ersten Anschein wenig werthaltig sind, weshalb er sein Portmonnaie hierfür besonders ungern öffnet (Simon/Dolan 1997).

\subsection{Ohne Wahrnehmung keine Wertschätzung}

Warum ist das so? Ein wesentlicher Grund ist das Wahrnehmungsproblem: Wertschätzung folgt aus Wahrnehmung. Die Immaterialität der Dienstleistung bewirkt, dass der Kunde das „Produkt“ nicht vor dem Kauf anfassen und prïfen kann (Meffert/Bruhn 1997). Daher haben Softwareanbieter - sie vermarkten ja eine „gefrorene Dienstleistung“ - ihr Produkt für die Präsentation im Handel aufdringlich sichtbar gestaltet: große Verpackungsformate umhïllen winzige Disketten. Ein bekannter Hersteller von Computer-Peripherie folgt diesem Gedanken: Der Wartungsvertrag für die Hardware, fruiher ein Zwei-Seiten-Papier aus einer Schublade unter dem Ladentisch, wird heute in einer voluminösen bunten Schachtel in das Regal gestellt. Die Bereitschaft der Kunden, für den After-Sales-Service Geld auf den Tisch zu legen, ließ sich so nachweislich steigern. Das dicke Service-Scheckheft symbolisiert einen Leistungsanspruch besser als die karge Rechnung, die den Servicevertrag wirksam werden lässt. Das teure Manager-Seminar schließt den überdimensionierten Ordner für die Dokumentation mit ein, die der Seminarteilnehmer beruhigt nach Hause tragen und dort über Jahre im Schrank verstauben lassen darf - immerhin hält er etwas in Händen fuir sein Geld.

Eine solche künstlich geschaffene Sichtbarkeit der an sich unsichtbaren Serviceleistung erleichtert es dem Kunden, über die psychologische Hürde des Dienstleistungskaufes zu springen - dass man nämlich knisterndes Geld hingeben soll, ohne dafuir eine wahrnehmbare, greifbare Sache zu bekommen. Das kann ja wohl, so das unterschwellige Gefuihl, kein fairer Tausch sein.
Dienstleister werden im Lichte dieser Erkenntnis ohne große Mühe neue Wege zur Erhöhung der kundenseitigen Zahlungsbereitschaft entdecken: Wo liegen bei unseren Leistungsangeboten Wahrnehmungsschranken? Wie machen wir unseren Service sichtbar oder anfassbar? Wie können wir dem Kunden Symbole der Leistungsqualität zeigen oder in die Hand geben?

\section{Drei Dimensionen der Dienstleistung}

\subsection{Potenzial, Prozess, Ergebnis}

Die Steigerung der Wahrnehmbarkeit ist eine wertvolle flankierende Maßnahme der Preisdurchsetzung, bildet jedoch nur einen ersten Ansatz zur Ertragssteigerung. Die zentralen Probleme der Preisgestaltung und Preisdurchsetzung im Service sind erst zu lösen, wenn man die unterschiedlichen Formen der Dienstleistung eingehender analysiert. Es wird keine optimale Vorgehensweise für „die Dienstleistung“ geben, sondern nur geeignete Strategien für bestimmte Typen der Serviceerstellung. Fachleute des Service Management unterscheiden zwischen drei Grundformen:

- potenzialorientierter,

- prozessorientierter und

- ergebnisorientierter Dienstleistung.

Die Potenzialdimension ist sichtbar vor, die Prozessdimension während der Leistungserstellung, die Güte der Ergebnisdimension erschließt sich hingegen erst danach. Die meisten Services, die uns im Alltag begegnen, sind Mischformen, wobei üblicherweise eine der drei Komponenten stark überwiegt (Bruhn/Stauss 2000).

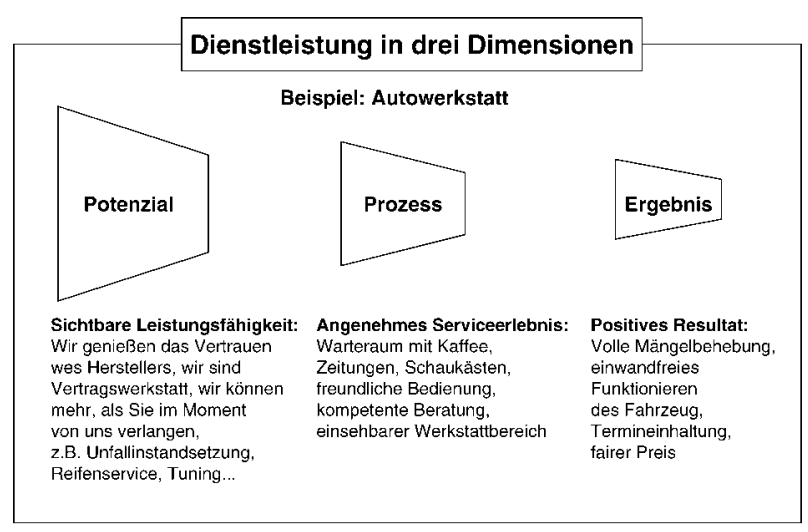

Abb. 2: Drei Dimensionen

\subsection{Nutzenargumention bei potenzialorientierten Services}

Es gibt Serviceangebote, bei denen der Kunde keinen für ihn im Hier und Jetzt erkennbaren (und im Nutzen berechenbaren) Vorteil erwirbt, sondern nur das Recht auf eine Aktion des Unternehmens bei Eintritt vorab definierter Fallsituationen. Das klassische Beispiel für diese potenzialorientierten Dienstleistungen bilden Versicherungen, Wachdienste oder Notrufzentralen. 
Ein Wartungsvertrag, der gegen Zahlung einer Jahrespauschale vorbeugende Instandhaltung eines Gerätes, schnelle Reparatur im Notfall plus Bereitstellung eines Ersatzgerätes bei Ausfall zusichert, gehört ebenfalls in diese Kategorie. Es liegt auf der Hand, dass ein derartiges Serviceangebot für das anbietende Unternehmen Kosten für die Vorhaltung von Reparaturkapazitäten mit sich bringt. Wie aber ist der Kunde zu bewegen, für einen derartigen Vertrag in die Tasche zu greifen? Die Schrecken des Geräteausfalls bilden für den Käufer noch eine ziemlich abstrakt Bedrohung - man kann sie zudem schlecht konkretisieren, wenn man ihm gerade das Gerät verkaufen möchte.

Ein wichtiges Verkaufsargument für die potenzialorientierte Dienstleistung liegt darin, dass der Nutzer nicht erst im (unwahrscheinlichen) Fall der Inanspruchnahme profitiert, sondern bereits durch das Vorhandensein des Anspruchs. Wer sein Haus gegen Feuer versichert, genießt das Gefuihl des Schutzes, selbst wenn ihn der Feuerteufel verschont: Er schläft besser. Der Haken aus Sicht der Anbieter versicherungsähnlicher Leistungen liegt darin, dass das Verhältnis zwischen Kosten (Beispiel: Zahlung für jährlichen Wartungsvertrag) und Nutzen im Schadensfall (hier: Reparaturkosten) aus dem Stand heraus kaum so überzeugend argumentiert werden kann wie bei der Feuerversicherung.

Dennoch lassen sich vom Grundsatz her nach dem gleichen Modell andere potenzialorientierte Services mit passenden Nutzenargumentationen unterlegen. „Mit diesem Wartungsvertrag garantieren wir, dass im Reparaturfall nach spätestens zwei Stunden der Techniker vor Ort ist!“ Das überzeugt den verantwortlichen Werksleiter natürlich erst, wenn er das Katstrophenszenario des Maschinenausfalls - Produktionsstillstand, Lieferverzögerung, Schlüsselkunde springt ab, Chef tobt! - im Kopf präsent hat. Dementsprechend ist ihm ein Nutzengefühl bereits fuir den Nicht-Schadensfall zu vermitteln: „Bei Abschluss unseres Wartungsvertrages können Sie Ihrem Chef immer nachweisen, dass Sie alles in Ihrer Macht Stehende tun, um Produktionsausfälle zu vermeiden!“ Auf diesem Argumentationsfundament wurden schon Weltkonzerne gebaut.

\subsection{Service erleben - die Prozessqualität}

Bei etlichen Services entsteht der Nutzen für den Kunden direkt aus der Beiwohnung des Service-Ereignisses, etwa im Freizeitbereich bei Theater-, Show- und Zirkusdarbietungen. Im Reise- und Touristiksektor leben viele Unternehmen ebenfalls vom Erlebniseffekt im Serviceprozess. Die Zahlungsbereitschaft der Kunden ist bei diesen prozessorientierten Dienstleistungen im Einzelfall hoch, aber starken Schwankungen unterworfen.

Die kundenseitige Wertschätzung ist bei prozessorientierten (man kann auch sagen: erlebnisorientierten) Dienstleistungen subjekiv geprägt, eine rationale Abwägung des Preis-Leistungs-Verhältnisses findet in geringerem Umfang statt als bei potenzial- oder ergebnisorientierten. Der Kunde weiß zudem erst hinterher, ob er für sein Geld den von ihm erhofften Gegenwert erhält nicht zufällig wird gerade bei diesen Services stets Vorkasse verlangt. Heranbildung von Stammkunden und Erzeugung von positivem Empfehlungsverhalten sind die wesentlichen Unterstuitzungspfeiler für die Preisdurchsetzung (Dehr/Biermann 1996).

Oft wird unterstellt, die Prozess- oder Erlebnisdimension sei ausschließlich bei Dienstleistungen für private Abnehmer relevant, doch finden wir auch im Business-toBusiness-Sektor Ansätze, dem Kunden für sein Geld neben der Basisleistung „eine gute Show“ zu liefern und ihn damit für eine Rechnungsstellung zugänglicher zu machen. Einige Kurierdienste und Speditionen erlauben es ihren Kunden, per Internet den Weg ihrer Sendung in Echtzeit selbst zu verfolgen. Das gibt diesem subjektiv ein gutes Gefuihl, weil er stets wissen kann, wo sich sein wertvolles Paket gerade befindet. Objektiv hilft es ihm hingegen wenig, wenn er online verfolgen kann, wie sein Container in Singapur ins Hafenbecken stuirzt.

\subsection{Wenn nur das Ergebnis zählt}

Die dritte Ebene nach Potenzial- und Prozessdimension ist die Ergebnisdimension. Hier interessiert allein das Resultat der Leistungserbringung. Pünktliche Auslieferung kennzeichnet die Leistungsguite der Spedition, der Sieg im aussichtslos scheinenden Gerichtsverfahren die des Anwalts, korrekte Ausfuihrung der komplizierten Auslandsuiberweisung die der Bank. Das ist sicher die wichtigste der drei Dimensionen: Wer bessere Ergebnisse liefert als der Wettbewerber, wird auf Dauer überlegen sein. Diese Auffassung ist verbreitet und ohne Zweifel korrekt, aber nicht durchgängig von hoher Praxisrelevanz. Die objektiven Leistungsunterschiede sind nämlich in vielen Branchen mittlerweile vernachlässigbar gering geworden. Die drei oder vier Konkurrenten, die sich um das selbe Marktsegment balgen, unterscheiden sich kaum noch durch fass- und messbare Unterschiede - und bei gleicher Ergebnisqualität schafft nur die Erlebnisqualität noch einen Konkurrenzvorteil.

Allerdings gibt es durchaus primär ergebnisorientierte Dienstleistungen, hier ist die erfolgsabhängige Honorierung eine auf der Hand liegende Alternative zu vorab vereinbarten Fixpreisen (Biermann 1999). Der Erfolg wird leicht feststellbar sein. Allenfalls der Ergebnisbeitrag des Dienstleisters im Vergleich zum Beitrag des Kunden selbst fuihrt zum Disput. Wo der Kunde zur Bezahlung hoher Verguitungen im garantierten Erfolgsfall bereit ist, erfreuen sich im Servicesektor erfolgsbasierte Vergütungssysteme wie Service-Garantien und Service Level Agreements (SLA) zunehmender Beliebtheit:

- bei der Servicegarantie wird die Erfuillung einer oder mehrerer Qualitätsmessgrößen verbindlich zugesagt, bei Nicht-Erfüllung erhält der Kunde den Kaufpreis ganz oder teilweise zurïck (z. B. Auslieferungszeitpunkt bei Kurierdiensten);

- im SLA werden verschiedene Kategorien der Leistungsgüte definiert, von hervorragend bis gerade noch akzeptabel; je nach Erfuillungsgrad wird die Ver- 
guitung dann im Nachgang innerhalb vorher festgelegter Bandbreiten berechnet.

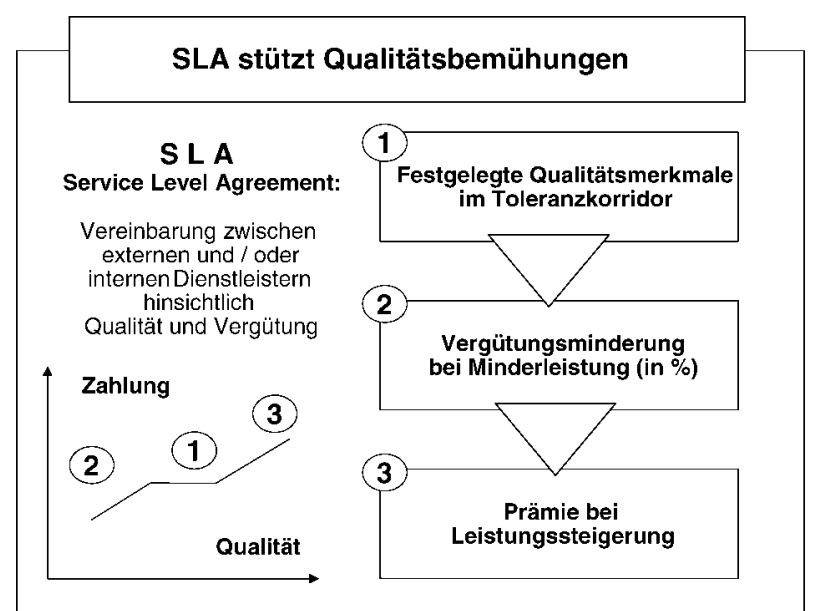

Abb. 3: Service Level Agreement

Das Service Level Agreement richtet die Aufmerksamkeit auf die Leistungsqualität und stellt eine klare Beziehung zwischen dieser Qualität und dem Leistungspreis her. Populär sind derartige Modelle in der Datenverarbeitung oder bei Wartungsverträgen, wo sich etwa Verfügbarkeitszeiten oder Reaktionszeiten der Servicetechniker bei Problemfällen als verguitungsrelevante Qualitätsparameter festlegen lassen.

\section{Leistungsdefinition in drei Dimensionen}

\subsection{Dreieinigkeit im Service}

In der Realität hat praktisch jede Leistung Potenzial-, Prozess- und Ergebniselemente. Mein Zahnarzt ist „mein“ Zahnarzt, weil:

- ich weiß, dass ich ihn auch kurzfristig im Notfall belästigen darf (Potenzial),

- die Prozedur bei ihm professionell und mit erkennbarem Bemühen um Schmerzfreiheit erfolgt (Prozess),

- man ihn nach gegluickter Behandlung lange nicht mehr besuchen muss (Ergebnis).

Die meisten traditionellen Dienstleister praktizieren diese Dreieinigkeit des Serviceangebotes mit erheblichem Geschick, ohne die Terminologie von Potenzial, Prozess und Ergebnis zu kennen. Man achte beim nächsten Besuch einer Anwaltskanzlei auf die Diplome und Ehrenurkunden an der Wand hinter dem Schreibtisch (Potenzial), das zum Verweilen einladende Ambiente und die Kompetenz ausstrahlende Gesprächsfuihrung des Rechtsgelehrten (Prozess) sowie die unauffälligen Symbole des Erfolgs in früheren Verfahren (Ergebnis).

Ein anderes Beispiel: Eine Hochschule

- verweist mit eindrucksvollen Gebäuden und dem Forscherdrang ihrer Professoren auf ihr Potenzial,

- unterstreicht die Prozessdimension im Lernerlebnis durch Hinweis auf angenehm kleine Studentengruppen sowie das fröhliche Campus-Ambiente,
- dokumentiert Ergebnisqualität durch statistischen Nachweis niedriger Studienabbrecherzahlen beziehungsweise hoher Erfolgsquoten ihrer Absolventen im Bewerbermarkt.

Technische Dienstleister, die ja meist einem industriellen Umfeld entstammen, beherrschen dagegen diese Klaviatur nur unvollständig, sie glauben, eine ordentliche Maschinenreparatur spricht für sich selbst. Tut sie auch - so lange man der einzige ist, der sie vollfuihren kann. Wo man aber im Wettbewerb zu anderen leistungsgleichen Anbietern steht, werden die Potenzialund Prozesselemente eine zunehmend wichtige Rolle spielen müssen.

\subsection{Leistungswahrnehmung und Erfolg}

Viele Serviceanbieter stehen vor dem Problem, dass sie durch gute Ergebnisse kaum noch glänzen können diese sind mittlerweile zum Branchenstandard geworden. In unseren Projekten begegnen uns regelmäßig Hersteller von Fahrzeugen oder Geräten, die uiber Jahre mit ihrem Werksservice gute Ergebnisse eingefahren haben, weil die (durchaus vorhandenen) freien Werkstätten objektiv klar unterlegen waren. Jetzt aber hat diese Konkurrenz aufgeholt und bietet gleiche Leistung zu deutlich niedrigerem Preis. Nun ist die Betonung der Potenzialdimension gefordert, es gilt deutlich zu machen, dass gerade in schwierigen Fällen der Hersteller weiter die höchste Servicekompetenz hat. Gleichzeitig muss die Prozessqualität angehoben werden (Meffert/ Bruhn 1995).

Vor diesem Hintergrund sind neue Produkt- und Preisgestaltungsmodelle zu diskutieren, die bewusst eine neben dem Kernangebot stehende Dimension der Dienstleistung in den Vordergrund stellen. Es gilt, die Kundenwahrnehmung der Leistungsgüte im Wettbewerbsvergleich und damit die Zahlungsbereitschaft zu erhöhen.

Ein Wachdienst kann beispielsweise durch martialische Uniformen, auffällig lackierte Fahrzeuge, sichtbar veranstaltete Notfalluibungen seiner Leute der ursprünglich potenzialorientierten Kernleistung „Bewachung“ eine Prozessdimension hinzufuigen. Das steigert den „Unterhaltungswert“ für den Kunden. Er kann überdies mit Quartalsberichten über Zahl und Art der abgewiesenen Gefahren (drei Einbrecher verjagt, einen Schwelbrand erkannt, zwei eingeschlossene Mitarbeiter befreit...) eine in der Kundenwahrnehmung bislang untergegangene Ergebnisdimension deutlicher herausstellen.

\section{Fazit}

Viele Unternehmen beklagen die niedrigen Gewinnmargen in ihrer Branche. Die Zukunft wird den Unternehmen gehören, die alle Gestaltungsmöglichkeiten auf der Produkt- und der Preisebene ausloten und realisieren. Alle - das heißt auch die der Kommunikation des Kunden- 
nutzens. Hier ist für viele noch Handlungsbedarf zu sehen - und Handlungsspielraum. Innovation besteht nicht allein im Erfinden neuartiger Produkte und Dienstleistungen, sondern auch im kreativen Einsatz verschiedener Marketinginstrumente. Die selektive Ausrichtung aller Serviceangebote auf für den Kunden erkennbare Wertschöpfung, das Sichtbar-Machen eigener Stärken und die Förderung der Zahlungsbereitschaft mittels einer überzeugenden Nutzenargumentation für den Kunden sind die neuen strategischen Erfolgsfaktoren im Service.

\section{Literaturhinweise}

Biermann, Thomas: Dienstleistungs-Management, München Wien 1999

Biermann, Thomas: Organisatorischer Wandel, in: Bankakademie (Hrsg.): Kompendium Management in Banking \& Finance, Frankfurt/Main 2002

Biermann, Thomas: Kundenbindung durch Innovation; in: Bruhn, Manfred/Stauss, Bernd (Hrsg.): Dienstleistungsmanagement Jahrbuch 2000, Wiesbaden 2000

Biermann, Thomas: Dienstleister müssen besser werden; in: Harvard Business Manager 2

Bruhn, Manfred/Stauss, Bernd (Hrsg.): Dienstleistungsqualität, Konzepte - Methoden - Erfahrungen, 3. Auflage, Wiesbaden 2000

Bruhn, Manfred/Homburg, Christian (Hrsg.): Handbuch Kundenbindungsmanagement, Wiesbaden 1998

Dehr, Gunter/Biermann, Thomas: Marketing-Management, München - Wien 1998

Dehr, Gunter/Biermann, Thomas (Hrsg.): Kurswechsel Richtung Kunde - die Praxis der Kundenorientierung, Frankfurt/ Main 1996

Halfmann, Marion/Laker, Michael: Preise gestalten, Planegg 1998

Hart, C./Heskett, J./Sasser, W. E.: The Profitable Art of Service Recovery, in: Harvard Business Review Jul-Aug 1990

Kamiske, Gerd. F. (Hrsg.): Rentabel durch TQM, Berlin - Heidelberg - New York 1996

Meffert, Heribert/Bruhn, Manfred: Dienstleistungsmarketing, Wiesbaden 1995

Simon, Hermann/Dolan, Robert: Profit durch Power Pricing, Frankfurt - New York 1997

\section{Autor}

Professor Dr. rer. pol. Thomas Biermann

Technische Fachhochschule Wildau

Fachbereich Betriebswirtschaft/Wirtschaftsinformatik

Tel. (0 33 75) 508-329 oder

Tel. (0 30) 8548831 (Service Management Institut)

E-Mail: SMIBerlin@aol.com 